九州大学学術情報リポジトリ

Kyushu University Institutional Repository

\title{
Controlled-Release Pesticides : 1. Dithiocarbamate Derivatives
}

Naruse, Hidetoshi

Laboratory of Pesticide Chemistry, Faculty of Agriculture, Kyushu University

Maekawa, Kazuyuki

Laboratory of Pesticide Chemistry, Faculty of Agriculture, Kyushu University

https://doi.org/10.5109/23491

出版情報：九州大学大学院農学研究院紀要. 21 (2/3)，pp.107-116，1977-03. Kyushu University バージョン：

権利関係 : 


\title{
Controlled-R elease Pesticides
}

\section{Dithiocarbamate Derivatives}

\author{
Hidetoshi Naruse and Kazuyuki Maekawa \\ Laboratory of Pesticide Chemistry, Faculty of Agriculture, \\ Kyushu University 46-02, Fukuoka 812 \\ (Received November 24, 1976)
}

\begin{abstract}
Poly(ethyleneimine $S$-alkyldithiocatbamate)s were prepared from poly(ethyleneimine sodium dithiocarbamate) and alkyl chlorides, aralkyl chlorides, substituted alkyl esters of chloroacetic acid, $\beta$-chloroethyl esters of carbamic and carboxylic acids, and $\mathrm{N}$-substituted chlorocarboxamides, respectively. The poly(ethyleneimine) derivatives thus obtained were decomposed physically and chemically to release sulfur and sulfur-containing substances, and acted as controlled-release herbicides or fungicides.
\end{abstract}

\section{INTRODUCTION}

There are a lot of papers on the chemical modification of synthetic and natural polymers in the medicinal field (Ascoli et al., 1967; Bichowsky-Slomnicki et al., 1956 ; Cornel and Donaruma, 1965 ; Dumitru et al., 1973). But, pesticidal polymers for controlled-release have not been studied well. Lately, roach tape (Plant, 1974), and others containing insecticides such as diazinon or chlorpyrifos in synthetic polymers were manufactured as controlled-release insecticides. On the other hand, controlled-release pesticides which can release active substances by chemical decomposition have been investigated by Allan et al. (1971) and others (Volkober and Varga, 1963). Beasley and Collins (1970) prepared controlled-release pesticide with a salt linkage. The authors prepared poly(ethyleneimine $S$-alkyldithiocarbamate)s which might be gradually decomposed in the field to give pesticidally active substances. To our knowledge, this is the first report on controlled-release pesticides which are polymers bound covalently with bioactive group.

\section{MATERIALS AND METHODS}

\section{Preparation of alkyl chloride derivatives}

$\beta$-Chloroethyl derivatives shown in Table 1 were synthesized by known methods. $\beta$-Chloroethyl $N$-alkyl or arylcarbamate was obtained from the reaction of alkyl or aryl isocyanate and ethylene chlorohydrin in dry tetrahydrofuran. The $\beta$-chloroethyl ester of 1,1-dichloropropionic acid and that of 2,4-dichlorophenoxyacetic acid were prepared from 1,1-dichloropropionyl or 2,4-dichlorophenoxyacetyl chloride and ethylene chlorohydrin in a dry tetrahydrofuran-pyridine mixture. $N$-( $3^{\prime}, 4^{\prime}$-dichlorophenyl)chloropropionamide was prepared from 3,4 - 
Table 1. $\beta$-Chloroethyl derivatives $\mathrm{RCH}_{2}-\mathrm{CH}_{2}-\mathrm{Cl}$.

\begin{tabular}{|c|c|c|c|c|c|c|}
\hline $\begin{array}{l}\text { Compd. } \\
\text { No. }\end{array}$ & $\mathrm{R}$ & $\begin{array}{l}\text { Yield } \\
(\%)\end{array}$ & $\begin{array}{c}\text { M.p.,b.p.* } \\
\text { (“C) }\end{array}$ & $\begin{array}{l}\mathrm{UV} \lambda_{\max }^{\mathrm{MeOH}} \\
\operatorname{nm}(\varepsilon)\end{array}$ & $\operatorname{IR} \nu_{\max }^{\mathrm{CHCl}_{3}}\left(\mathrm{~cm}^{-1}\right)$ & References \\
\hline 1 & Q-NHCOO- & 83 & $47 / \mathrm{THF}$ & $\begin{array}{l}280(120) \\
234(3310)\end{array}$ & 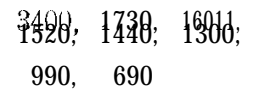 & $\begin{array}{l}\text { Sprinson } \\
\text { (1941) }\end{array}$ \\
\hline$\because$ & $\mathrm{HCOO}$ & 90 & 46/THF & $\begin{array}{l}276(220) \\
239(3360)\end{array}$ & $\begin{array}{lll}3488, & 29528, & 17200 \\
1270, & 1090, & 640\end{array}$ & $\begin{array}{l}\text { McKey and } \\
\text { Braun (1.951) }\end{array}$ \\
\hline 3 & NHCOO- & 68 & $101 / \mathrm{C}_{6} \mathrm{H}_{6}$ & $\begin{array}{l}290(1410) \\
223(11490)\end{array}$ & $\begin{array}{ccc}3400 & 3050, & 1730 \\
1530 & 1490, & 1340: \\
1190 . & 1110 & \end{array}$ & $\begin{array}{l}\text { McKey and } \\
\text { Braun (1951) }\end{array}$ \\
\hline 4 & $\mathrm{CH}_{3} \mathrm{NHCOO}^{-}$ & 31 & $108 / 7 \mathrm{~mm}^{*}$ & $205(80)$ & 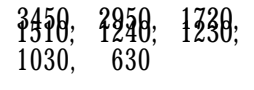 & $\begin{array}{l}\text { Sprinson } \\
\text { (1941) }\end{array}$ \\
\hline 5 & $-\mathrm{NHCO}$ & 76 & $92 / \mathrm{C}_{6} \mathrm{H}_{6}$ & $213(5030)$ & $\begin{array}{ccc}3400 & 3000 & 1680 \\
15980 & 15108 ; & 14880 \\
650 & & \end{array}$ & $\operatorname{Good}(1961)$ \\
\hline 6 & $\mathrm{Cr} \overbrace{-}^{\mathrm{Cl}}-\mathrm{OCH}_{2} \mathrm{COO}$ & 16 & $171-6 / 7 \mathrm{~mm}^{*}$ & $\begin{array}{l}283(2270) \\
228(8490) \\
222(8440)\end{array}$ & $\begin{array}{lll}2900, & 1760, & 1480, \\
1430, & 1300, & 1180, \\
1648, & 1090, & 870,\end{array}$ & $\begin{array}{l}\text { McNew and } \\
\text { Hoffmann } \\
(1950)\end{array}$ \\
\hline 7 & $\mathrm{CH}_{3} \mathrm{CCl}_{2} \mathrm{COO}$ & 21 & $60-1 / 35 \mathrm{~mm}^{*}$ & $206(443)$ & $\begin{array}{l}2958, \quad 1758, \quad 1488, \\
1000, \quad 900, \quad 650\end{array}$ & $\begin{array}{l}\text { Scheuerer et } \\
\text { al. }(1960)\end{array}$ \\
\hline
\end{tabular}

dichloroaniline and $\beta$-chloropropionyl chloride at the molar ratio of $2: 1$ in dry benzene.

Chloromethyl derivatives shown in Table 2 were obtained on usual processes. $N$ - $\left(3^{\prime}, 4^{\prime}\right.$-dichlorophenyl) or alkyl chloroacetamide was synthesized from chloroacetyl chloride and 3,4-dichloroaniline or alkylamine at the molar ratio of $1: 2$ in dry benzene. 2,4-Dinitro-5-methyl-6-phenyl chloroacetate was prepared from sodium 2,4-dinitro-5-methyl-6-phenolate and chloroacetyl chloride in dry acetone.

$2 \cdot(p$-Chlorophenoxy) ethyl chloroacetate was synthesized as follows ; sodium 2-( $p$-chlorophenoxy)ethylate was prepared from $25 \mathrm{~g}$ of 2 -( $p$-chlorophenoxy)ethanol and $3.3 \mathrm{~g}$ of metallic sodium in $200 \mathrm{ml}$ of dry benzene. Then, $16.2 \mathrm{~g}$ of chloroacetyl chloride was gradually added to the ethylate suspension at $0^{\circ}-5^{\circ} \mathrm{C}$, and the mixture was further stirred for 7 hours at room temperature avoiding moisture. Colorless precipitates formed were removed from the reaction mixture. The filtrate treated with activated carbon was evaporated under reduced pressure to give two oily layers. The lower layer was separated, washed with n-hexane and distilled at $160^{\circ}-164^{\circ} \mathrm{C}(0.2 \mathrm{~mm} \mathrm{Hg})$ to give yellow oil. Anal. Found (\%): $\mathrm{C}$, 49.00; $\mathrm{H}, 4.13$. Calcd. (\%) for $\mathrm{C}_{10} \mathrm{H}_{10} \mathrm{O}_{3} \mathrm{CI}_{3}: \mathrm{C}, 48.88 ; \mathrm{H}, 4.08$. Yield $31 \%$. 
Table 2. Chloromethyl derivatives $\mathrm{RCOCH}_{2}-\mathrm{Cl}$.

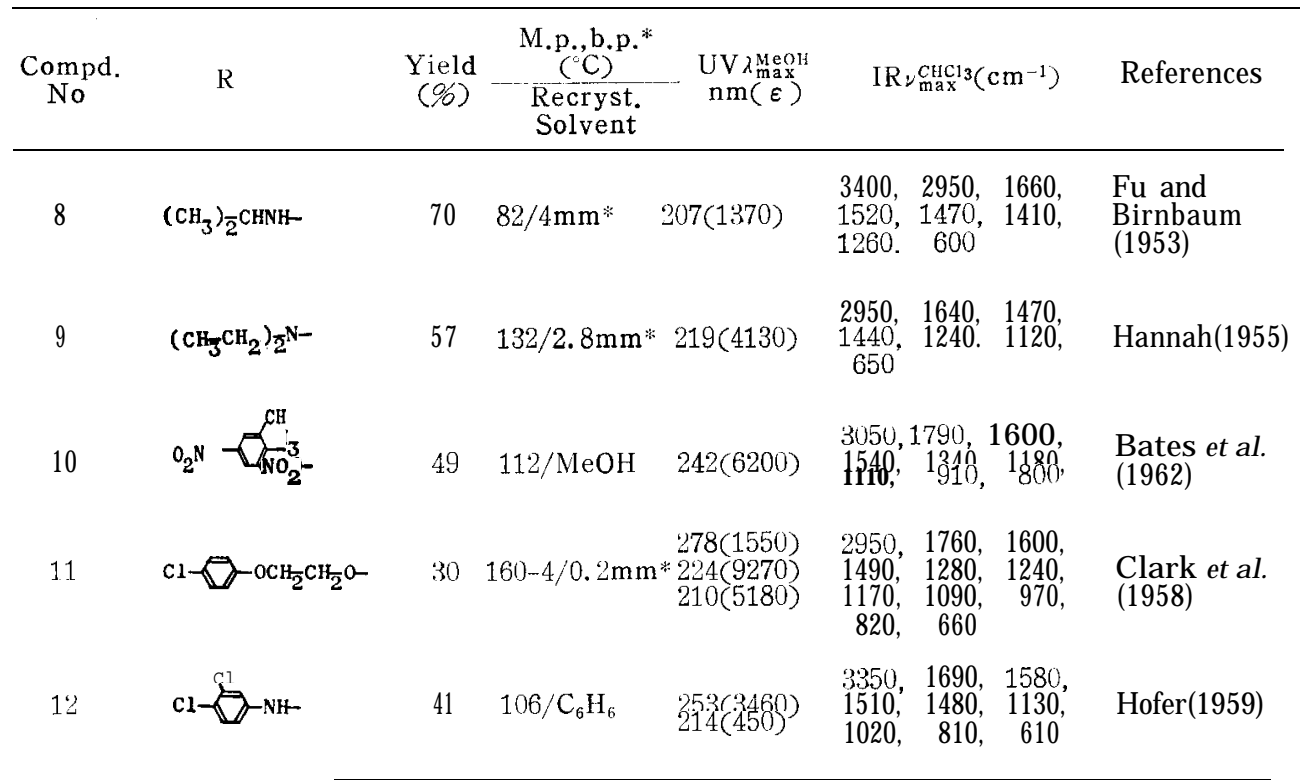

\section{Synthesis of poly(ethyleneimine S-alkyldithiocarbamate) $\mathbf{S}$}

Twenty two poly(ethyleneimine $S$-alkyldithiocarbamate)s were prepared, as shown in Scheme 1, from poly(ethylencimine sodium dithiocarbamate) and alkyl chlorides, benzyl chlorides, alkyl chloroacetates, and chloromethyl or $\beta$ chloroethyl derivatives containing pesticidal groups listed in Table 1 and 2, respectively. The reaction were carried out by heating the starting materials in dimethylformamide for several hours at $50^{\circ} \mathrm{C}$ with stirring. The isolation of the modified polymers was performed by precipitation (by adopting dimethylformamide-methanol, tetrahydrofuran, chloroform, ether and so on) in the addition of a small amount of saturated sodium chloride solution. The properties of these polymers obtained were shown in Table 3 .

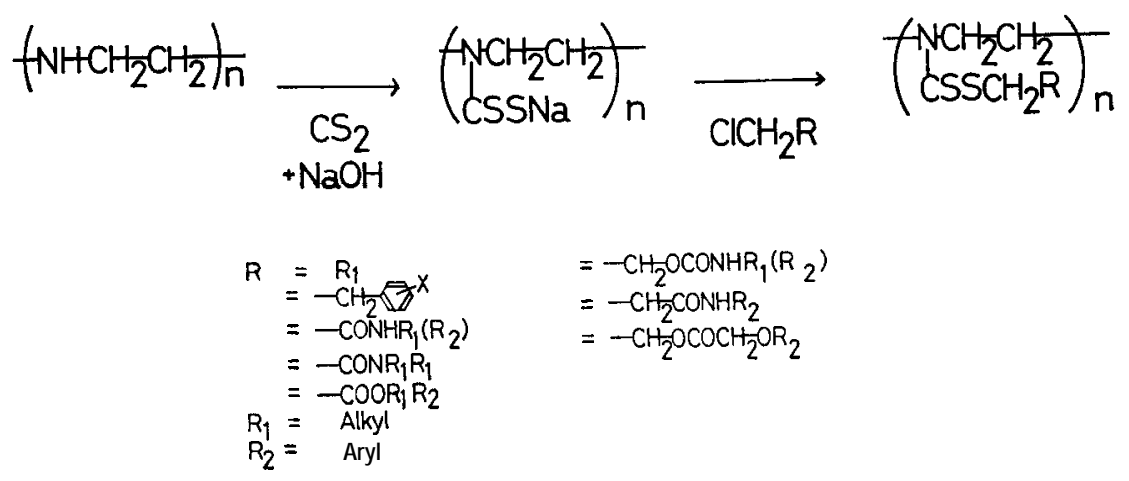

Scheme 1. General synthetic methods of polymers. 


\begin{tabular}{|c|c|c|c|c|c|c|}
\hline \multirow{2}{*}{$\begin{array}{l}\text { Compd. } \\
\text { No. }\end{array}$} & \multirow[t]{2}{*}{$\mathrm{R}$} & \multirow{2}{*}{$\begin{array}{c}\mathrm{UV} \\
\lambda_{\max }^{\mathrm{DMF}}(\mathrm{nm})\end{array}$} & \multirow{2}{*}{\multicolumn{2}{|c|}{$\frac{\mathrm{IR}}{\boldsymbol{\nu}_{\max }^{\mathrm{KBr}}\left(\mathrm{cm}^{-1}\right)}$}} & \multicolumn{2}{|c|}{$\begin{array}{l}\text { Analysis (\%) } \\
\text { Found }\end{array}$} \\
\hline & & & & & $\overline{\mathrm{N}}$ & Ash \\
\hline 13 & $\left(\mathrm{CH}_{3}\right)_{\overline{2}} \mathrm{CH}$ & 281 & $\begin{array}{l}3350, \\
1360,\end{array}$ & $\begin{array}{l}2900,1670,1480 \\
1220,1110,980\end{array}$ & 11.99 & $\because$ \\
\hline 14 & $\mathrm{CH}_{2}=\mathrm{CHCH}_{2}$ & 282 & $\begin{array}{l}3050 \\
1400\end{array}$ & $\begin{array}{l}2900,1480,1470, \\
1210,1130,980\end{array}$ & 11.67 & \\
\hline 15 & $\mathrm{HOCH}_{2} \mathrm{CH}_{\frac{2}{2}}$ & 281 & $\begin{array}{l}3150 \\
1460\end{array}$ & 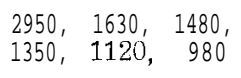 & 11.19 & + \\
\hline 16 & $\mathrm{CH}_{3} \mathrm{CH}_{2} \mathrm{OOCCH}_{\overline{2}}$ & 288 & $\begin{array}{l}3359 \\
1440 \\
1150\end{array}$ & $\begin{array}{l}2950,1730,1480, \\
1360,1290,1210, \\
1020\end{array}$ & 9.67 & \\
\hline 17 & $\mathrm{CH}_{2}=\mathrm{CHCH}_{2} \mathrm{OOCCH}-$ & 288 & $\begin{array}{l}3350 \\
1480 \\
1130\end{array}$ & 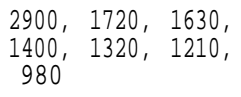 & 9.70 & \\
\hline 38 & & 279 & $\begin{array}{l}2950 \\
1400 \\
860\end{array}$ & $\begin{array}{l}1600,1510,1480, \\
1340,1110,990,\end{array}$ & 9.73 & \\
\hline 19 & $\partial-\mathrm{CH}_{2}$ & 280 & $\begin{array}{l}3150 \\
1090\end{array}$ & $\begin{array}{l}1480,1400,1210, \\
1020,830\end{array}$ & 7.96 & \\
\hline 20 & & 279 & $\begin{array}{l}3150 \\
1400 \\
990\end{array}$ & $\begin{array}{l}2900, \quad 1470, \quad 1460, \\
1210,\end{array}$ & 8.02 & \\
\hline 21 & $\partial \mathrm{CH}_{2}$ & 275 & $\begin{array}{l}3150 \\
1220 \\
820\end{array}$ & $\begin{array}{l}2900,1480,1400, \\
1110,1030,990,\end{array}$ & 8.44 & \\
\hline 22 & & 281 & $\begin{array}{r}3050 \\
1480 \\
980\end{array}$ & $\begin{array}{l}2900,1680,1600, \\
1450,1280,1200,\end{array}$ & 7.98 & \\
\hline 23 & & 281 & $\begin{array}{l}3200 \\
1370 \\
820\end{array}$ & $\begin{array}{l}2900,1580,1490, \\
1280,1130,1080,\end{array}$ & 12.09 & \\
\hline 34 & $\mathrm{COOCH}_{2} \mathrm{CH}_{2}$ & 284 & $\begin{array}{l}3350 \\
3570 \\
1290\end{array}$ & $\begin{array}{l}2900,1720,1660, \\
1510,1480,1350, \\
1140,990,800\end{array}$ & 15.20 & \\
\hline 25 & & 279 & $\begin{array}{l}3250 \\
1600 \\
1070\end{array}$ & $\begin{array}{l}\text { 2950, 1720, 1650, } \\
1480,1440,1310,\end{array}$ & 15.81 & -1 \\
\hline
\end{tabular}


Table 3. (continued)

\begin{tabular}{|c|c|c|c|c|c|}
\hline \multirow{2}{*}{$\begin{array}{l}\text { Compd. } \\
\text { No. }\end{array}$} & \multirow[t]{2}{*}{$\mathbf{R}$} & \multirow{2}{*}{$\underset{\lambda_{\max }^{\mathrm{DMV}}(\mathrm{nm})}{\mathrm{UV}}$} & \multirow{2}{*}{$\frac{\mathrm{IR}}{\nu_{\max }^{\mathrm{KBr}}\left(\mathrm{cm}^{-1}\right)}$} & \multicolumn{2}{|c|}{$\begin{array}{c}\text { Analysis (\%) } \\
\text { Found }\end{array}$} \\
\hline & & & & $\mathrm{N}$ & Ásh \\
\hline 26 & $8-\mathrm{NHCOOCH}_{2} \mathrm{CH}_{2}$ & 278 & $\begin{array}{l}3200,2900,1720,1660 \\
1590,1480,1210,1160 \\
770\end{array}$ & 16.23 & \\
\hline 27 & $\mathrm{CH}_{3} \mathrm{NHCOOCH}_{2} \mathrm{CH}_{2}$ & 283 & $\begin{array}{l}3300,2950,1710,1480 \\
1440,1240,1120,980\end{array}$ & 16.22 & $H$ \\
\hline 28 & $\left(\mathrm{CH}_{3}\right)_{\overline{2}} \mathrm{CHNHCOCH}_{2}$ & 281 & $\begin{array}{l}3250,2950,2100,1640 \\
1530,1360,1160,980\end{array}$ & 13.57 & \\
\hline 29 & $\left(\mathrm{CH}_{3} \mathrm{CH}_{2}\right)_{2} \mathrm{NCOCH}_{2}$ & 283 & 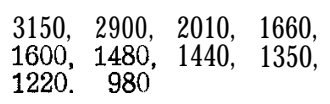 & 13.81 & \\
\hline 30 & & 282 & $\begin{array}{l}3200,2900,1650,1580 \\
1480,1370,1230,1120 \\
1020,870\end{array}$ & 12.97 & \\
\hline 31 & $\mathrm{FO}_{2} \mathrm{OOCCH}$ & 283 & $\begin{array}{l}3200,2900,2100,1710 \\
1650,1480,1320,1210 \\
1130,980,880\end{array}$ & 13.03 & \\
\hline 32 & $-\mathrm{OCH}_{\overline{2}} \mathrm{CH}_{\overline{2}} \mathrm{OOCCH}_{\overline{2}}$ & 285 & $\begin{array}{l}2950,1730,1600,1480 \\
1410,1290,1240,1150 \\
1060,990,820\end{array}$ & 7.81 & \\
\hline 33 & $\mathrm{Cl}-\stackrel{\mathrm{Cl}}{2}-\mathrm{OCH}_{2} \mathrm{COOCH}_{2} \mathrm{CH}_{2}$ & 288 & $\begin{array}{l}3200,2900,1650,1480 \\
1430,1210,1110,1010 \\
830\end{array}$ & 13.88 & 1 \\
\hline 34 & $\mathrm{CH}_{3} \mathrm{CCl}_{2} \mathrm{COOCH}_{2} \mathrm{CH}_{2}$ & 280 & $\begin{array}{l}3300,2950,1720,1480 \\
1400,1220,1210,3140 \\
1010\end{array}$ & 13.22 & \\
\hline
\end{tabular}

Poly(ethyleneimine sodium dithiocarbamate) was prepared from poly(ethyleneimine) (Tokyokasei Organic Chemicals), sodium hydroxide, and carbon disulfide (Okawara et al., 1966). $\eta_{s p} / c\left(0.5 \%\right.$ aq. soln., $\left.20^{\circ} \mathrm{C}\right)=0$. 3830. Anal. Found (\%): $\mathrm{C}, 26.40 ; \mathrm{H}, 5.21 ; \mathrm{N}, 10.44$. UV $\lambda_{\max }^{\mathrm{H}_{2} \mathrm{O}}(\mathrm{nm}): 292(=\mathrm{N}-\stackrel{\mathrm{C}}{\mathrm{C}}=\mathrm{S}), 257(-\mathrm{CSSNa}), \mathrm{UV}$ $\lambda_{\max }^{\mathrm{DMF}}(\mathrm{nm}): 298(=\mathrm{N}-\mathrm{C}=\mathrm{S}) . \quad$ IR $\nu_{\max }^{\mathrm{KBr}}\left(\mathrm{cm}^{-1}\right): 3400-3000(-\mathrm{NH}-$, hydrate $-\mathrm{OH})$, $2900\left(-\mathrm{CH}_{2}-\right), 1630$ (hydrate $\left.-\mathrm{OH}\right), 1460(=\mathrm{N}-\stackrel{\mathrm{I}}{\mathrm{C}}=\mathrm{S}), 1120(\mathrm{C}=\mathrm{S})$ (Fig. 1-B).

The content of dithiocarbamoyl group was determined by LJV spectrometry (12.4 mole \%) and by the method of Clark et al. (Clark et al., 1951) (14.3 mole $\%)$ or A. 0. A. C.'s method (Horwitz, 1975) (17.5 mole \%).

Poly (ethyleneimine $S$-[2-( $p$-chlorophenoxy) ethoxycarboxymethyl] dithiocarbamate] was synthesized as follows; when $50 \mathrm{ml}$ of $10 \% 2$-( $p$-chlorophenoxy)ethyl chloroacetate solution in dimethylformamide were added to $100 \mathrm{ml}$ of $5 \%$ 
poly(ethyleneimine sodium dithiocarbamate) dimethylformamide solution, the mixture reacted exothermally resulting an orange solution (Braun und Engelbertz, 1923 ; Okawara et al., 1966). The colored solution was stirred for 6 hours at $60^{\circ} \mathrm{C}$ to deposit sodium chloride and others. The reaction mixture cooled to room temperature was filtered off to separate the insoluble substances. The filtrate poured into 1.51 of methanol was salted out with $10 \mathrm{ml}$ of saturated sodium chloride aqueous solution to give orange precipitates. The precipitates were washed with water, methanol, and ether, then dried under reduced pressure. Yield 4.2 g. UV $\lambda_{\max }^{\mathrm{DMF}}(\mathrm{nm}): 285(=\mathrm{N}-\stackrel{\mathrm{C}}{\mathrm{C}}=\mathrm{S}), \mathrm{IR} \quad \nu_{\max }^{\mathrm{KRr}}\left(\mathrm{cm}^{-1}\right): 3350(-\mathrm{NH}-)$, 2900, $1410\left(-\mathrm{CH}_{2}-\right), 1720\left(-\mathrm{COOCH}_{2}-\right), 1490,1470(-\mathrm{CH}=\mathrm{CH}-,=\mathrm{N}-\stackrel{\mathrm{C}}{\mathrm{C}}=\mathrm{S}), 1280$ $-1240\left(\mathrm{Cl}-<=>-\mathrm{OCH}_{2}-\right), 1150\left(-\mathrm{COOCH}_{2}-\right), 1110(\mathrm{C}=\mathrm{S}), \$ 20$ (2 substituted $\left.\mathrm{Cl}-\mathrm{O}_{-}\right)($Fig. 1-A).

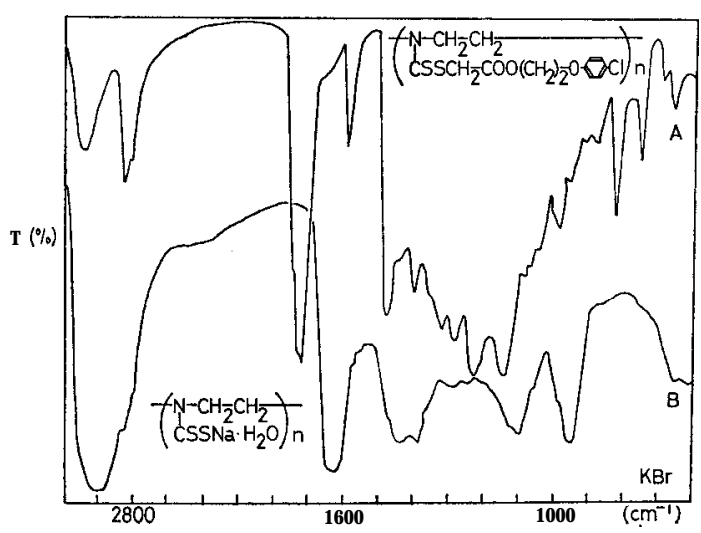

Fig. 1. IR spectra of poly\{ethyleneimine S- [2-( $p$-chlorophenoxy)ethoxycarbonylmethyl] dithiocarbamate) (A) and poly(ethyleneimine sodium dithiocarbamate) (B).

\section{Degradation of poly(ethyleneimine sodium or $S$-alkyldithiocarbamate)s by natural light}

Dimethylformamide solution of poly(ethyleneimine sodium dithiocarbamate) and poly (ethyleneimine $S$-alkyldithiocarbamate)s were exposed to natural light in the door at room temperature for about 20 days to give a turbid solution and precipitates, and to release sulfur, some sulfur-containing substances and others. The decomposition products of p-methylbenzyl compound (21), for example, were detected by TLC (silicagel $\mathrm{G}$, acetone, $\mathrm{PdCl}_{2}$ or $\mathrm{I}_{2}$ ) as 7 spots.

\section{PESTICIDAL BIOASSAY}

\section{Herbicidal test}

Forty grams of soil (weak acidic upland soil of Kyushu University Farm, $\mathrm{pH}$ 6.2) and $10 \mathrm{ml}$ of water were mixed with a $0.2 \%$ poly(ethyleneimine $S$-alkyldi- 
thiocarbamate). Twenty seeds of radish (Raphanus sativus L. var. radicular Dc.) and barnyard millet (Panicum crusgalli L.) were respectively put in the treated soil. These plants grew under light keeping wet. The temperature was kept at $25^{\circ}-28^{\circ} \mathrm{C}$ for radish and $30^{\circ}-34^{\circ} \mathrm{C}$ for barnyard millet at $70-80 \%$ humidity. The medium of barnyard millet was made wetter after germination. The herbicidal activity was defined as the average of growth damage (\%) and germination (\%) (Blackman, 1951; Ready and Grant, 1947; Sugawara and Koyama, 1971). The results are shown in Table 4.

Table 4. Herbicidal activities of poly(ethyleneimine S-alkyldithiocarbamate)s

\begin{tabular}{|c|c|c|c|c|c|c|}
\hline \multirow{3}{*}{$\begin{array}{c}\text { Compd. } \\
\text { No. }\end{array}$} & \multicolumn{3}{|c|}{ Radish } & \multicolumn{3}{|c|}{ Barnyard millet } \\
\hline & \multirow{2}{*}{$\begin{array}{l}\text { Germi- } \\
\text { nation } \\
(\%)\end{array}$} & \multicolumn{2}{|c|}{ Damage $(\%)$} & \multirow{2}{*}{$\begin{array}{c}\text { Germi- } \\
\text { nation } \\
(\%)\end{array}$} & \multicolumn{2}{|c|}{ Damage $(\%)$} \\
\hline & & $19 \mathrm{~d}$ & $22 \mathrm{~d}$ & & $19 \mathrm{~d}$ & $22 \mathrm{~d}$ \\
\hline Control & $8 \overline{3}$ & 0 & 6 & 95 & 32 & 63 \\
\hline $\begin{array}{l}\text { Positive } \\
\text { control \# }\end{array}$ & 0 & 100 & & 0 & 100 & \\
\hline 13 & 50 & 20 & 20 & 95 & 26 & 73 \\
\hline 14 & 40 & 13 & 13 & 90 & 28 & 72 \\
\hline 15 & $85^{\text {*** }}$ & 0 & 0 & 85 & 94 & 100 \\
\hline 16 & $60 *$ & 5 & 33 & 95 & 0 & 0 \\
\hline 17 & 85 & 0 & 6 & 95 & 5 & 10 \\
\hline 18 & 65 & 8 & 46 & 95 & 21 & 26 \\
\hline 19 & 85 & 6 & 12 & 95 & 0 & 5 \\
\hline 20 & 100 & 20 & 25 & 95 & 18 & 18 \\
\hline 21 & 85 & 0 & 6 & 85 & 6 & 12 \\
\hline 22 & 85 & 6 & 6 & 95 & 11 & 11 \\
\hline 23 & 80 & 6 & 13 & 85 & 6 & 12 \\
\hline 24 & 90 & 6 & 11 & 95 & 79 & 94 \\
\hline 25 & 55 & 18 & 18 & 95 & 95 & 100 \\
\hline 26 & 45 & 11 & 11. & 95 & 89 & 100 \\
\hline 27 & 35 & 57 & 57 & 100 & 25 & 65 \\
\hline 28 & 70 & 14 & 14 & 100 & 20 & 75 \\
\hline 29 & 75 & 7 & 7 & 90 & 0 & 11 \\
\hline 30 & 50 & U & 0 & 90 & 83 & 100 \\
\hline 31 & 5 & 0 & 0 & 95 & 63 & 100 \\
\hline 32 & 70 & 79 & 93 & 75 & 20 & 53 \\
\hline 33 & 20 & 100 & 100 & 90 & 61 & 78 \\
\hline 34 & 65 & 46 & 67 & 90 & 100 & 100 \\
\hline
\end{tabular}

$\# \mathrm{CH}_{3}-\mathrm{CCl}_{2}-\mathrm{COOC} \mathrm{H}_{2}-\mathrm{CH}_{2}-\mathrm{Cl}$ Seedling growth: * repression, ** elongation.

\section{Antifungal test}

Disc paper method using the filter papers (Toyokagaku Qualitative No. 2) with $6 \mathrm{~mm}$ diameter was employed for the fungicidal test of the polymer $(0.5$ $\%$ dimethylformamidc solution) or their light-decomposed materials on Aspergillus niger, Trichoderma viride and Pythium debaryanum. Asp. niger, T. viride and P. debaryanum were cultured on $3 \%$ Czapek-agar and potato-sucrose-agar, respectively at $28^{\circ} \mathrm{C}$. The antifungal activity shown in Table 5 was qualitatively evaluated from the ratio of sporulation, inhibiting zone and inhibitory persistence (Golden and Oster, 1947; Sugawara and Koyama, 1971). 
Table 5. Antifungal activity of poly(ethyleneimine $S$-alkyldithiocarbamate)s and their decomposed products by light.

\begin{tabular}{|c|c|c|c|c|c|c|}
\hline \multirow{2}{*}{$\begin{array}{l}\text { Compd. } \\
\text { No. }\end{array}$} & \multicolumn{2}{|c|}{$\begin{array}{l}\text { Aspergillus } \\
\text { niger }\end{array}$} & \multicolumn{2}{|c|}{$\begin{array}{c}\text { Trichoderma } \\
\text { viride }\end{array}$} & \multicolumn{2}{|c|}{$\begin{array}{c}\text { Pythium } \\
\text { debaryanum }\end{array}$} \\
\hline & Intact & Decompd.* & Intact & Decompd.* & Intact & Decompd.* \\
\hline DMF & \pm & & \pm & & \pm & \\
\hline $\mathrm{CH}_{3}-\mathrm{NHCSSNa}$ & HI & & H & & $H$ & \\
\hline $\begin{array}{l}13 \\
14 \\
16 \\
18 \\
20 \\
21 \\
22 \\
23 \\
24 \\
28 \\
29 \\
30 \\
32 \\
33 \\
34 \\
35\end{array}$ & $\begin{array}{l} \pm \\
\pm \\
\pm \\
\pm \\
\pm \\
\pm \\
\pm \\
\pm \\
\pm \\
\pm \\
\pm \\
\pm \\
\pm \\
\pm \\
\pm \\
t\end{array}$ & $\begin{array}{c} \pm \\
+ \\
+ \\
+ \\
+ \\
\pm \\
\pm \\
\pm \\
\pm \\
\pm \\
\pm \\
\pm \\
\pm \\
\pm \\
\pm \\
H\end{array}$ & $\begin{array}{l} \pm \\
\pm \\
\pm \\
\pm \\
\pm \\
\pm \\
\pm \\
\pm \\
\pm \\
\pm \\
\pm \\
\pm \\
\pm \\
\pm \\
\pm\end{array}$ & $\begin{array}{l} \pm \\
\pm \\
+ \\
\frac{1}{1} \\
+ \\
\frac{1}{ \pm} \\
\pm \\
\pm \\
\pm \\
\pm+1 \\
\pm \\
\pm \\
\pm \\
\pm \\
\pm\end{array}$ & $\begin{array}{l} \pm \\
\pm \\
\pm \\
\pm \\
\pm \\
\pm \\
\pm \\
\pm \\
\pm \\
\pm \\
\pm \\
\pm \\
\pm \\
\pm \\
\pm \\
\pm t\end{array}$ & 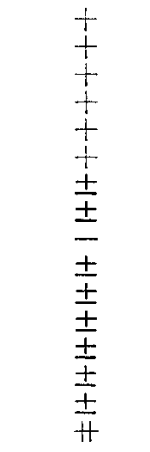 \\
\hline
\end{tabular}

Inhibition zone was determined at five to eleven days after inoculation.

HIt zone of inhibition was more than $2 \mathrm{~cm}$,

$\mathrm{HI}$ zone of inhibition was more than $1 \mathrm{~cm}$,

$H$ zone of inhibition was less than $1 \mathrm{~cm}$,

+ organism failed to grow on disc,

\pm slight growth of organism on disc,

$\rightarrow$ no inhibition.

(35) poly(ethyleneimine sodium dithiocarbamate)

* Conditions, see text.

\section{RESULTS AND DISCUSSION}

Judging from these experimental data, poly(ethyleneimine sodium or $S$-alkyldithiocarbamate) is thought to be a copolymer with about 15 mole $\%$ of dithiocarbamate which decomposes easily to release some substances of lower molecular weight.

$S$-ethane-2-ol (15) damaged strongly the growth of barnyard millet for 19 days and S-arylcarbamates $(24,25,26)$ also showed a considerable inhibition on barnyard millet growth. $S$ - $\left(3^{\prime}, 4^{\prime}\right.$-dichlorophenyl)carbamoylethyl (30) and 2,4 dinitro-5-methyl-6-phenyl S-acetate (31) exhibited remarkable selective activity on barnyard millet for a relatively long period. On the other hand, 2-( $p$-chlorophenoxy)ethyl S-acetate ('32) and S-ethyl 2,4-dichlorophenoxyacetate (33) depressed the growth of radish seedling. S-ethyl 1,1-dichloropropionate (34) was herbicidally effective on the growth of barnyard millet. Considering from these data, some derivatives of poly(ethyleneimine S-alkyldithiocarbamate) whose carbon-sulfur and nitrogen-carbon linkages of dithiocarbamate group can be cleaved by hydrolysis and light might be useful as a controlled-release herbicide. 
Poly(ethyleneimine) inhibited the growth of Asp. niger and T. viride. In addition, water-soluble poly(ethyleneimine sodium dithiocarbamate) (35) was more effective for the inhibition of the growth than poly(ethyleneimine) was. The poly(ethyleneimine sodium dithiocarbamate) decomposed by natural light at room temperature had more inhibitory effect on Asp. niger than that of the intact polymer and sodium methyldithiocarbamate. In this case, the lower molecular antifungal compounds released from poly(ethyleneimine sodium dithiocarbamate) were assumed to be sulfur, sodium hydrosulfide, and carbonyl sulfide.

The poly(ethyleneimine S-alkyldithiocarbamate)s could not suppress the fungus growth in the dark for several days. But, the poly(ethyleneimine $S$. alkyldithiocarbamate)s (solution of dimethylformamide) decomposed by natural light were effective antifungally as shown in $S$-allyl(14), S-acetate (16), S-mchlorobenzyl (20), and others. The released substances are still unknown except sulfur, but some sulfur-containing compounds like mono or disulfide derivatives are to be thought. On the whole, these facts suggested that the poly(ethyleneimine sodium dithiocarbamate) and poly(ethyleneimine $S$-alkyldithiocarbamate)s were chemically and physically decomposed to release antifungal substances.

\section{ACKNOWLEDGEMENT}

We wish to express our gratitude to Associate Professor Dr. Morifusa Eto, for helpful discussions.

\section{REFERENCES}

Allan, G. G., C. S. Chopra, A. N. Neogu and R. M. Wilkins 1971 Controlled-release pesticide part II, the synthesis of herbicide-forest solid waste combination. Tappi, 54: 1293-1294

Ascoli, F., G. Casini, M. Ferappi and E. Tubara 1967 A polymeric nitrofuran derivative with a prolong antibacterial action. J. Med. Chem., 10: 98-99

Bates, A. N., D. M. Spencer and R. L. Wain 1962 Fungicides V. The fungicidal properties of 2-methyl-4,6-dinitrophenol (DNC) and some of its esters. Ann. Appl. Biol., 50: 21-32

Beasley, M. J. and R. L. Collins 1970 Water-degradable polymers for controlled release of herbicides and other agent. Science, 169: 769-770

Bichowsky-Slomnicki, L., A. Berger, J. Kurtz and E. Katchalski 1956 The antibacterial action of some basic amino acid copolymers. Arch. Biochem. Biophys., 65: 400-413

Blackman, G. E. 1951 Herbicides and selective phytotoxicity. Ann. Rev. Plant Physiof., 2: $199-230$

Braun, J. V. und P. Engelbertz 1923 Gemischte organische Sulfide und Bromcyan. Chem. Ber., 56: 1573-1577

Clark, D. G., H. Bauw, E. L. Stanley and W. F. Hester 1951 Determination of dithiocarbamates. Anal. Chem., 23: 1842-1846

Clark, R. J., A. Isaacs and J. Walker 1958 Derivatives of 3, 4-xylidine and related compounds as inhibitors of influenza, relationships between chemical structure and biological activity, J. Pharmacol., (Brit.), 13: 424-435

Cornel, R. J. and L. G. Donaruma 1965 Poly-2-methacryloxytropone, a synthetic biological active polymer. J. Polymer Sci., A3: 827-828 
Dumitru, S., R. Burnaru und C. R. Simionescu 1973 Bioaktive Polymer I, Bioaktive Derivate der Zellulose. Cellulose Chemistry and Technology, 7: 553-562

Fu, S.-C. J. and S. M. Birnbaum 1953 The hydrolytic action of acylase I on N-acylamino acids. J. Am. Chem. Soc., 75; 918-920

Golden, M. J. and K. A. Oster 1947 Evaluation of a fungicidal laboratory test method. $J$. Am. Pharm. Assoc., 36: 283-288

Good. N. E. 1961 Inhibitors for the Hill reaction. Plant Physiol., 36: 788-803

Hannah, L. H. 1955 Field studies with a new class of herbicidal chemicals. Proc. Northeast Weed Control Conf., pp. 15-19 (Chem. Abstr., 49: 6529)

Hofer, K. 1959 Esters as dying assistants. Appl. Oct., 19: 4

Horwitz, W. 1975 Official Methods of Analysis of the Association of Official Analytical Chemists. 12th Ed. Benjamin Franklin Station, Washington, pp. 117-118

McKey, A. F. and R. 0. Braun 1951 Cyclization of 2-chloroethyl substituted ammonocarbonic acids. J. Org. Chem., 16: 1829-1834

McNew, G. L. and 0. L. Hoffmann 1950 Growth-regulant, herbicidal, and physical properties of 2,4-D and related compounds. Iowa State Coll. J. Sci., 24: 189-208 (Chem. Abstr., $44:$ 9105-9106)

Okawara, M., M. Ori, T. Nakai and E. Imoto 1966 Dithiocarbamate-ki o fukumu poly (ethyleneimine) no gosei to hanno. Kogyo Kagaku Zasshi, 69: 766-771

Plant, A. F. 1974 Controlled-release pesticides attract interest. Chem. Eng. News, 52(39): 20-23

Ready, D. and V. W. Grant 1947 A rapid selective method for determination of low concentrations of 2,4-dichlorophenoxyacetic acid in aqueous solutions. Bot.Gaz., 109: 39-44

Scheuerer, G., H. Pommer, H. Strummeyer and A. Fischer 1960 Esters of chlorinated carboxylic acids and their herbicidal compositions. Ger. Appl. Sept., 24: 3

Sprinson, D. B. 1941 Synthesis of N-substituted chlorine carbamates and trimethyl( $\beta$-phenylaminoethyl) ammonium chloride. J. Am. Chem. Soc., 63: 2249-2251

Sugawara, H. and K. Koyama 1971 Noyaku no Seibutsukenteiho. Nankodo, Tokyo, pp. 164

Volkober, Z. and L. S. Varga 1963 Synthesis of Macromolecular herbicide. Vyscomol. Soed., 5: 139-144 